\title{
Implementación de la metodología de pares para estimar el consumo de drogas lícitas e ilícitas
}

\author{
JORGE RODRIGUEZ(1), ENRIQUE HERNÁNDEZ(1), MIGUEL CUMSILLE(1)
}

\section{RESUMEN}

Este estudio describe la metodología de pares y los resultados obtenidos del consumo de drogas en población universitaria. La metodología de pares está orientada a la detección de poblaciones en que se supone podria haber una sub declaración (poblaciones ocultas), un tipo de estas poblaciones son los consumidores de drogas, en especial las ilegales. El objetivo es obtener la prevalencia del consumo de drogas legales e ilegales a través de la metodologia de pares, que consiste en que un sujeto que es el entrevistador, es reconocido por otro sujeto que es el entrevistado como un "PAR". El sujeto entrevistador debe ser previamente capacitado en el instrumento y su aplicación. Se obtuvo una muestra aleatoria de 142 jovenes universitarios, entre 18 y 26 años, de ambos sexos. La tasa de consumo de los últimos 30 dias para cualquier tipo de droga licita alcanzó a 92,3\%, siendo similares las tasas de consumo de alcohol y cigarrillos 80,3\% y 78,9\%, respectivamente. Para las drogas ilicitas fue de 63,4\%, donde el $100 \%$ declara consumir marihuana. Las tasas de consumo de cocaina y éxtasis resultaron de 4,9\% y 2,1\%, respectivamente. Puesto que la mayoría de los indices obtenidos no son semejantes a los que resultan a través de los métodos tradicionales de recolección de información, parece aconsejable seguir evaluando la metodología propuesta.

Palabras claves: Metodologia de pares, poblaciones ocultas, estudiantes universitarios, consumo de drogas ilicitas, consumo de alcohol y cigarrillos.

\section{ABSTRACT \\ IMPLEMENTATION OF THE PEERS METHODOLOGY TO ESTIMATE THE CONSUMPTION OF LICIT AND ILLICIT DRUGS}

In this study the peer methodology is described and the results obtained from drug consumption in a university population. The peer methodology is directed toward the detection of populations in which supposedly there could be a subdeclaration (hidden populations); one type of these populations are the drug consumers, specially of illegal drugs. The objective is that of obtaining the prevalence of legal and illegal drug consumption through the peer methodology which consists in that one subject, the interviewer, is recognized by another subject, the respondent, as a "PEER". The interviewer must previously have been trained in the instrument and its application. A random sample of 142 young university students of both sexes, with ages ranging from 18 to 26. was obtained. The consumption rate for any type of licit drug during the last 30 days reached $92.2 \%$, the rates of alcohol and cigarrette consumption being similar, $80.3 \%$ and $78.9 \%$

(1) Escuela de Salud Pública. Facultad de Medicina. Universidad de Chile jrodrigu(emed.uchiled 
respectively. For illicit drugs, the consumption was $63.4 \%$, of which $100 \%$ declared consuming marihuana. The consumption rates for cocaine and extasis resulted being $4.9 \%$ and $2.1 \%$, respectively. In view of the fact that the major part of the rates obtained are not similar to those resulting from traditional methods of information gathering, it seems advisable to continue evaluating the proposed methodology.

Key words: Peer methodology; hidden populations; university students; illicit drug consumption; alcohol and cigarrette consumption.

\section{INTRODUCCIÓN}

Los países de América Latina están cada día más preocupados del problema de la drogadicción, ya que cada día existen más jóvenes drogadictos!.

En el último periodo, el uso y abuso como el tipo de droga se han convertido en un problema social que afecta la estabilidad de todas las instituciones de una sociedad, en especial las sociales y políticas ${ }^{1-4}$.

En Chile, se ha realizado un gran número de trabajos que aborda el tema, lo que muestra su magnitud. En especial se ha dedicado a ello el Consejo Nacional para el Control de Estupefacientes, Conace, el cual realiza estudios a nivel nacional, regional y comunal, para evaluar la magnitud del problema en población general y escolar ${ }^{5-12}$. Estos y otros antecedentes, reflejan que el problema de la drogadicción y sus consecuencias en el mundo y, en particular, en nuestro país están lejos de estar resueltos.

Unido a lo anterior y al fenómeno de la drogadicción ilícita, se encuentra el problema de la drogadicción lícita, fundamentalmente relacionado con alcoholismo, que afecta cada vez con mayor fuerza a la población juvenil.

Conace publicó recientemente los resultados de la quinta encuesta escolar de drogas, realizada en noviembre del 2003 , que muestra una disminución en el consumo de drogas en escolares, con relación al año 2001. Los resultados se ven influenciados por la caida del consumo de la marihuana, que de $14,5 \%$ descendió a 12,9\%, medida en escolares de octavo básico a cuarto medio. No obstante lo positivo del resultado anterior, preocupa el hecho que el consumo de drogas más fuertes, como la cocaína, éxtasis y pasta base, no experimentó cambios significativos entre los periodos estudiados ${ }^{6-13}$.

En investigaciones realizadas fuera de la Región Metropolitana, cabe destacar los hallazgos hechos por Alvarado y col. en la XII Región de Magallanes, donde se estudió una muestra de 1.270 estudiantes de enseñanza media, de todos los colegios de la región, encontrándose que el 15,4\% de los encuestados había probado droga, siendo la marihuana la más frecuentemente utilizada, que representa un $66,2 \%$ en el grupo de consumidores que la han probado alguna vez en la vidalt

En poblaciones de jóvenes entre 19 y 25 años, los indices que se obtienen a partir de los entrevistados por Conace en drogas denominadas lícitas (alcohol y tabaco), son de 67 y $56,2 \%$, respectivamente; y bajan sustancialmente cuando se habla de drogas ilícitas que no alcanza al 9\% (marihuana con $8,3 \%$, cocaína con $1,9 \%$ y pasta base con $0,66 \%$ ).

Por otro lado, el análisis de la población de nivel socioeconómico medio-alto, demuestra que los índices son inferiores a la población de jóvenes de 19 a 25 años y disminuyen la declaración en un 10\%, llegando a un $61 \%$ para el alcohol y $41,5 \%$ para el cigarrillo. En el caso de las drogas ilícitas, los índices son de $2,6 \%$ (marihuana un 2,5\%, cocaina un $0,40 \mathrm{y}$ pasta base un $0,1 \%$ ). En la comuna de Las Condes, en este mismo grupo de edad los índices son mayores que los declarados a nivel nacional, llegando las drogas ilícitas a un $15,9 \%$ y las lícitas a un $87,9 \%$. El registro de la información desde el $1^{\circ}$ estudio realizado en 1994 hasta el del 2002, muestra que el consumo tanto a nivel de drogas lícitas e ilícitas está aumentando en este grupo de edad. Además, 
es necesario reconocer que uno de los grandes problemas frente a este tipo de información y que puede afectar los índices de consumo, es su sub declaración, en especial de las drogas ilícitas lo que hace mucho más preocupante el tema, en especial en los jóvenes de 19 a 25 años $^{8-12}$.

Existe una variedad de metodologías orientadas a ubicar a poblaciones donde existe una sub declaración, denominadas poblaciones ocultas (Fountain, J y Griffiths, P., 1999). Una de éstas, es el entrevistador de acceso privilegiado (Griffiths, P., Gossop, M., Powis, B., y Strang, J., 1993; Kuebler, D. y Hausser, D., 1997) $15-17$.

La magnitud e impacto que tiene el uso de drogas tanto ilícitas como lícitas en nuestro país, constituye como se dijo, un problema no resuelto que afecta al individuo, la familia y la sociedad en su conjunto ${ }^{2-5,8}$.

El objetivo de esta investigación es aportar más antecedentes relacionados con la magnitud del consumo de drogas en jóvenes universitarios pertenecientes a un nivel socio económico medio alto-alto de la ciudad de Santiago, aplicando una adaptación de la metodología entrevistadores de acceso privilegiado (EAP), que se denomina metodología de pares, con el propósito de disminuir la sub declaración del consumo y, por ende, mejorar los índices acerca del consumo de drogas lícitas e ilícitas.

\section{MATERIAL Y MÉTODO}

El estudio es de tipo exploratorio en cuanto a la metodología usada y correlacional. La población son jóvenes (18 a 26 años), universitarios, de nivel socio económico medio alto y alto (el nivel socioeconómico se asoció al valor de la mensualidad, sobre $\$ 240.000$, nivel medio-alto o alto), que asisten a una universidad privada de Santiago en el año 2003. Se utilizó la metodología de pares en la recolección de los datos que consiste en solicitar a las instituciones, alumnos voluntarios para hacer la entrevista. Se presentaron 12 voluntarios, 6 de cada institución, sin saber los objetivos del estudio, se realizaron dos reuniones de capacitación. En la $1^{\circ}$ sesión se explicó el objetivo del estudio, el instrumento a usar y la forma de aplicación, enfatizando el anonimato y privacidad que debería tener la entrevista. En la $2^{\circ}$, se aplicó el instrumento haciendo las veces de entrevistador y de entrevistado. Para minimizar posibles sesgos que pudieran afectar la sub declaración o sobre declaración del consumo de drogas, se asignó a los entrevistadores facultades distintas a la que pertenecían y se les dio la instrucción. La selección de la muestra se realizó considerando el listado de alumnos por facultad, respetando la proporcionalidad del género.

Se consideró drogas lícitas a tabaco y/o alcohol y se definió como fumador a aquel que consume al menos dos veces por semana; para el alcohol o marihuana que al menos consuma una vez por semana, y en el resto de las drogas (cocaína, éxtasis, pasta base u otra) que se consuma, al menos, una vez al mes.

Se estudiaron 142 jóvenes de dos instituciones privadas, que accedieron a realizar este tipo de estudio, considerando una tasa de consumo de un $24 \%$, con un nivel de confianza de un $95 \%$ y un error máximo de estimación de un 7\%.

En la recolección de la información se utilizó un instrumento especialmente para este estudio, basado en las preguntas que utiliza Conace, las que fueron de tipo cerrado.

La muestra estuvo conformada por un $60,6 \%$ de hombres, con edades de 21,5 años, con una desviación estándar de 1,9 años. Los entrevistadores fueron de ambos sexos y con edades entre 18 y 22 años.

Tabla 1. Tasa de consumo de drogas en estudiantes universitarios de 18 a 26 años de niveles sociocconómicos medio-alto y alto e intervalos de confianza del $95 \%$. Santiago, Año 2003

\begin{tabular}{lccc}
\hline $\begin{array}{l}\text { Caracte- } \\
\text { rísticas }\end{array}$ & $\begin{array}{c}\mathbf{N}^{\mathbf{0}} \text { de } \\
\text { consumidores }\end{array}$ & $\begin{array}{c}\% \text { de } \\
\text { consumo }\end{array}$ & $\begin{array}{c}\text { Intervalo de } \\
\text { Confianza } 95 \%\end{array}$ \\
\hline D. Licitas & 131 & 92,3 & 87,$92 ; 96,68$ \\
Tabaco & 112 & 78,9 & 72,$19 ; 85,61$ \\
Alcohol & 114 & 80,3 & 73,$76 ; 86,84$ \\
D. llicitas & 90 & 63,4 & 55,$48 ; 71,32$ \\
Marihuana & 90 & 63,4 & 55,$48 ; 71,32$ \\
Cocaina & 7 & 4,9 & 1,$35 ; 8,45$ \\
Pasta Base & 0 & 0,0 & \\
Extasis & 3 & 2,1 & 0,$00 ; 4,07^{*}$ \\
\hline
\end{tabular}

*: Intervalo unilateral. 
Para la descripción y análisis de datos se utilizó el programa estadístico SPSS y se obtuvieron indicadores descriptivos e intervalos de confianza del $95 \%$, en el caso de porcentajes muy extremos se utilizaron intervalos unilaterales.

\section{RESULTADOS}

Las drogas lícitas son las que presentan el mayor consumo con un $92,3 \%$, donde el alcohol

Tabla 2. Asociación entre el consumo de drogas lícitas e ilicitas en estudiantes universitarios de 18 a 26 años de niveles socioeconómicos medio-alto y alto. Santiago, Año 2003

\begin{tabular}{|c|c|c|c|c|}
\hline \multirow[b]{3}{*}{$\begin{array}{l}\text { Droga } \\
\text { lícita }\end{array}$} & \multicolumn{3}{|c|}{ Droga ilícita } & \multirow[b]{2}{*}{ TOTAL } \\
\hline & $S$ & SI & NO & \\
\hline & $\mathrm{N}^{0}$ & $\%$ & $\mathrm{~N}^{0} \%$ & $\mathrm{~N}^{0} \quad \%$ \\
\hline $\mathrm{SI}$ & 86 & 65,6 & $45 \quad 34,4$ & $131 \quad 92,3$ \\
\hline $\mathrm{NO}$ & 4 & 36,4 & 763,6 & $11 \quad 7,7$ \\
\hline Total & 90 & 63,4 & 5236,6 & 142100,0 \\
\hline
\end{tabular}

Tabla 3. Asociación entre el consumo de alcohol y tabaco en estudiantes universitarios de 18 a 26 años de niveles socioeconómicos medio-alto y alto. Santiago, Año 2003

\begin{tabular}{|c|c|c|c|c|c|c|}
\hline \multirow[b]{3}{*}{ Tabaco } & \multicolumn{4}{|c|}{ Alcohol } & \multirow{2}{*}{\multicolumn{2}{|c|}{ TOTAL }} \\
\hline & \multicolumn{2}{|c|}{ SI } & \multicolumn{2}{|c|}{ NO } & & \\
\hline & $N^{0}$ & $\%$ & $\mathbf{N}^{0}$ & $\%$ & & $\%$ \\
\hline $\mathrm{Sl}$ & 95 & 84,8 & 17 & 15,2 & 112 & 78,9 \\
\hline NO & 19 & 63,3 & 11 & 36,7 & 30 & 21,1 \\
\hline Total & 114 & 80,3 & 28 & 19,7 & 142 & 100,0 \\
\hline
\end{tabular}

es declarado por $80,3 \%$ y el consumo de tabaco por el 78,9\% de los jóvenes entrevistados. El consumo de drogas ilícitas es inferior al consumo de drogas lícitas como se observa en la Tabla 1, el consumo de drogas ilícitas alcanza a un $63,4 \%$. La declaración del consumo de marihuana también fue de un $63,4 \%$, es decir, todos los que informaron que consumían drogas ilícitas. En los otros tipos de drogas ilícitas el consumo disminuye notoriamente, donde la cocaina cae al 4,9\% y el éxtasis de un $2,1 \%$.

Los intervalos de confianza que se obtienen son preocupantes ya que en el caso de drogas lícitas, entre el $88 \%$ y el $97 \%$ de los jóvenes declara consumir algún tipo de drogas lícita y entre el $56 \%$ y $71 \%$ de los jóvenes declara consumir algún tipo de droga ilícita.

Se observó que el grupo de consumidores de drogas lícitas tiene un mayor porcentaje de consumidores de drogas ílicitas que el grupo que no consume drogas lícitas $(\mathrm{P}=0,0262)$, como se observa en la Tabla 2.

Se observó asociación entre el hábito de fumar y el consumo de alcohol, el porcentaje de fumadores que consume alcohol es significativamente mayor que en el grupo de no fumadores $(P=0,0178)$.

\section{DISCUSIÓN}

Los estudios nacionales e internacionales muestran que el consumo de drogas está concentrado en los grupos de edades más jóvenes (18 a 25 años). Chile es coincidente con esto, sin embargo, el consumo de drogas ilícitas se ubica en un rango ligeramente más amplio, de 14 a 35 años. Esto justifica que la preocupación de los estudios de drogas esté enfocada principalmente a estos grupos etáreos.

Los resultados de este estudio confirman la estructura del consumo de otros estudios, en especial los realizados por Conace, que muestran que el consumo de drogas licitas es superior al de drogas ilícitas. En éstos el mayor consumo de drogas licitas está concentrado en alcohol y el consumo de drogas ilícitas, en la marihuana.

Llama la atención los altos índices de consumo obtenidos para cualquier tipo de drogas con relación a los obtenidos por las metodologías tradicionales. En particular, al comparar con los estudios nacionales realizados por Conace para este mismo grupo de edad, se encuentra que los resultados de este estudio para alcoholismo son de un $80,3 \%$ en comparación con el $67 \%$.

En el caso de drogas ilícitas la situación es mucho más preocupante, ya que del $9 \%$ informado por Conace, en este estudio aumenta al $63,4 \%$ de los entrevistados, y la cocaína pasa del $1,9 \%$ informado por Conace a un $4,9 \%$, situación similar a la detectada por esta misma institución para comunas asociadas a 
niveles socioeconómicos medio alto-alto (Las Condes; Vitacura; La Reina; Providencia). Las asociaciones encontradas entre drogas lícitas e ilícitas, como también entre las drogas lícitas, coinciden con los otros estudios en este sentido.

Cabe preguntar, ¿los índices serán replicables a toda la población de jóvenes de estas edades? ¿los resultados obtenidos serán similares en otros grupos etáreos, género, nivel socio económico, regional, comunal o nacional, etc.? ¿Habrá algún sesgo en la declaración? Por ejemplo, podría ocurrir que el joven entrevistado no quiera sentirse distinto al estereotipo de joven arriesgado, violento, que no se detiene ante nada, y, por ello, produce una sobre declaración del consumo o, tal vez, que el joven reconozca al entrevistador como cercano a su entorno familiar o, quizás, que se sienta entregando información muy personal, produciendo una sub declaración.

De acuerdo a los resultados sobre el consumo de drogas en general, que muestran grandes diferencias en los índices anteriores, es que el estudio sugiere seguir evaluando esta nueva metodología en este mismo grupo, como en otros grupos de edad y en otros lugares, como así también una revisión de las metodologías tradicionales usadas en la recolección de datos en los estudios donde se puede prever que hay una sub declaración, población oculta.

\section{REFERENCIAS}

1.- OFICINA PANAMERICANA DE SALUD, Noticias e información del Centenario. www oas.org/ defaultesp.htm

2.- Mc WHINTER, FloRENZANO U. Correlatos psicosociales de la farmacología: Resultados de un estudio en Santiago de Chile. Rev Psiquiátrica 1998; $15(1): 10-22$.

3.- BOLETIN DE LA OFICINA SANITARIA PANAMERICANA DROGAS. Vol $107 \mathrm{n}^{\circ} 6 \mathrm{diC}$ 1989.

4.- Oea-ClCAD (Comisión internacional para el control del abuso de drogas). Estrategia en el Hemisferio.
Julio 2001 .

5.- CONSEJO NACIONAL PARA EL CONTROL DE ESTUPEFACIENTES (CONACE), Estudio del consumo de drogas en población escolar de Chile a nivel comunal, informe final, 1999.

6.- CONSEJO NACIONAL PARA EL CONTROL DE ESTUPEFACIENTES (CONACE). Estudio nacional de drogas en población escolar de Chile, 2001. Informe Nacional.

7.- CONSEJO NACIONAL PARA EL CONTROL DE ESTUPEFACIENTES (CONACE). Estudio nacional de drogas en población escolar de Chile, 2001. Informe Nacional.

8.- CONSEJO NACIONAL PARA EL CONTROL DE ESTUPEFACIENTES (CONACE). Quinto estudio nacional sobre drogas en población general de Chile. 2002. Santiago de Chile.

9.- CONSEJONACIONAL PARA EL CONTROL DE ESTUPEFACIENTES (CONACE). Quinto estudio nacional sobre drogas en población general de Chile. 2000. Santiago de Chile.

10.- CONSEJO NACIONAL PARA EL CONTROL DE ESTUPEFACIENTES (CONACE). Quinto estudio nacional sobre drogas en población general de Chile. 1998. Santiago de Chile.

11. - CONSEJO NACIONAL PARA EL CONTROL DE ESTUPEFACIENTES (CONACE). Quinto estudio nacional sobre drogas en población general de Chile. 1996. Santiago de Chile.

12.- CONSEJONACIONAL PARA EL CONTROL DE ESTUPEFACIENTES (CONACE), Quinto estudio nacional sobre drogas en población general de Chile. 1994. Santiago de Chile.

13.- ARANEDA J C, CUMSILLE F. Consumo de drogas en población escolar de Chile en alumnos de $8^{\circ}$ básico a $4^{\circ}$ medio, 2003. Rev Chil Salud Pública 2004; 8 (2): 63-71.

14.- ALVARADO R, NEVES L, VIDAL A. Factores asociados al consumo de drogas entre estudiantes de enseñanza media en la región de Magallanes. Rev Chil Salud Pública 2003; 7(3): 134-45.

15.- FOUNTAIN J, GRIFFITHS P. Synthesis of qualitative research on drug use in European Union: report on an EMCDDA proyect. European Addiction Research 1999; 5: 4-20.

16.- GRIFFITIS P, GOSSOP M, POWIS B, STRANG J. Reaching hidden populations of drug users by privileged access interviewers: methodological and practical issues. Addiction 1993; 88: 1617-26.

17.- KUEBLER D, HAUSSER D. The Swiss Hidden Population Study: practical and methodological aspects of dates collection by privileged access interviewers. Addiction 1997; $92: 325-34$.

Usted puede comentar éste y otros artículos publicados en la Revista Chilena de Salud Pública, enviando un correo electrónico a revistasp amed.uchile.cl 